\title{
Estrés como factor limitante en el proceso de toma de decisiones: una revisión desde las diferencias de género
}

\author{
Stress as a Limiting Factor in the Decision Making Process: A Review \\ from Gender Differences \\ Stress como fator limitante no processo de toma de decisões: uma revisão \\ desde as diferenças de gênero
}

\author{
Laura Patricia Cote Rangel, ${ }^{*}$ Andrea Milena García Becerra* \\ Universidad de La Sabana, Colombia
}

Doi: dx.doi.org/10.12804/ap134.1.2016.02

\section{Resumen}

En la presente revisión teórica se reportan diversos artículos que han mostrado la importancia de estudiar los efectos del estrés en la población y su relación con otros procesos cognitivos y patologías. Uno de los procesos que se ve afectado es la toma de decisiones, la cual - se ha mostrado - crea un círculo entre la presencia de estrés, que afecta neurofisiológicamente el sistema nervioso, y la toma de decisiones equivocadas. Así mismo, se ha encontrado que la respuesta en este proceso cognitivo ante situaciones potencialmente peligrosas se manifiesta de diferentes formas en hombres y mujeres con relación a los tiempos de respuesta, toma de decisiones más riesgosas y malas decisiones financieras.

Palabras clave: estrés; toma de decisiones; diferencias de género.

\begin{abstract}
In this theoretical review, several studies reported have shown the importance of studying the effects of stress levels in the population and its relation to other cognitive processes and pathologies, a processes that is the decision making process, which is positively associated with stress, so that is created a circle between stress that affect neurophysiologically the nervous system and bad decisions. Likewise, it has been found that the response in this cognitive processes and the presence of dangerous situations is manifested differently in men and women with regard to response times, making riskier decisions and bad financial decisions.

Keywords: stress; decision making; differences gender.
\end{abstract}

* Laura Patricia Cote Rangel y Andrea Milena García Becerra, Facultad de Psicología, Universidad de La Sabana, Chía, Colombia. La correspondencia relacionada con este artículo debe ser dirigida a Andrea Milena García Becerra, autopista norte, kilómetro 7, campus Universidad de La Sabana, Facultad de Psicología, Chía, Colombia. Correo electrónico: andreagb@unisabana.edu.co

Cómo citar este artículo: Cote, L. P. \& García, A. M. (2016). Estrés como factor limitante en el proceso de toma de decisiones: una revisión desde las diferencias de género. Avances en Psicología Latinoamericana, 34(1), 19-28. doi: dx.doi.org/10.12804/ apl34.1.2016.02 


\section{Resumo}

Na presente revisão teórica reportam-se diversos artigos que têm mostrado a importância de estuda os efeitos dos níveis de stress na população e sua relação com outros processos cognitivos e patologias, um destes processos que se vê afeitado é a tomada de decisões, a qual se tem demostrado que cria um círculo entre a presença de stress, que afeita neurofisiologicamente ao sistema nervoso e a toma de decisões equivocadas. Assim mesmo, tem-se encontrado que a resposta neste processo cognitivo ante situações potencialmente perigosas manifesta-se de diferentes formas em homens e mulheres com relação aos tempos de resposta, tomada de decisões mais arriscadas e más decisões financeiras. Palavras-chave: stress; tomada de decisões; diferenças de gênero.

Desde hace algunos años, el estudio del estrés ha tomado fuerza en el medio académico en múltiples disciplinas como la medicina, la salud ocupacional, la psicología e, incluso, ha tenido cabida en la economía y políticas públicas de las naciones. El estrés hace parte de la vida cotidiana y se considera una reacción normal y adaptativa del ser humano; sin embargo, en los últimos años el estrés ha pasado de ser una respuesta normal a ser objeto de investigaciones. Con mayor frecuencia se ha identificado cómo la presencia de estrés crónico es capaz de causar diversas problemáticas para el individuo, en la medida en que tiene un impacto directo y negativo en el funcionamiento y subsecuentemente en la calidad de vida de la persona (Wiener, 2011).

Según estadísticas de la American Psychological Association (2009), el 69\% de los empleados reporta tener un grado significativo de estrés en sus trabajos. Así mismo, el $41 \%$ de ellos manifiesta tensión durante toda la jornada laboral. La American Psychological Association (2012), en un estudio realizado con 1226 personas mayores de 18 años de edad, encontró que los adultos perci- ben que sus grados de estrés están en aumento. El $44 \%$ de los encuestados indicó que en los últimos 5 años su estrés aumentó considerablemente, y solo el 27\% reportó una reducción. Estos porcentajes se mantienen cuando se analiza el último año, cuando el 39\% asegura que se siente más estresado y tan solo el 17\% considera que el estrés se ha reducido. Finalmente, el estudio señala que el $53 \%$ de las personas refiere haber tenido o tener alguna enfermedad producto de índices elevados de estrés.

Thompson (2010) afirma que en Estados Unidos las compañías deben emplear más de 300 billones de dólares anuales en incapacidades, bajas de producción y gastos de seguridad social, producto del estrés en sus empleados, y se estima que más de 200 millones de personas toman algún medicamento para el estrés.

Estas cifras revelan que el estrés afecta a la población en general, y está relacionado con otros problemas de salud que causan muertes en América: enfermedades de corazón, cáncer y enfermedades cerebro-vasculares (Pan American Health Organization, 2013).

Además, el estrés está asociado con respuestas agresivas, el síndrome de burnout (desgaste profesional), depresión, frustración y negativamente con la capacidad de adaptabilidad o afrontamiento, desempeño laboral, memoria y la toma de decisiones (Leka, Griffiths \& Cox, 2004).

El presente trabajo tiene como propósito exponer el efecto que puede tener el estrés como factor limitante del proceso de toma de decisiones, al igual que la relación con estructuras cerebrales y su sustrato neurofisiológico. Adicionalmente, se pretende establecer si se presentan diferencias entre hombres y mujeres en la toma de decisiones en situaciones de estrés.

Para llevar a cabo la siguiente revisión teórica, se tuvieron en cuenta cuatro criterios de selección: (1) ninguna de las investigaciones realizadas debía tener más de 10 años de antigüedad; (2) para la selección de los artículos se utilizaron palabras 
clave como stress and decision making (DM), gender diferences and stress/DM y neural dynamics of stress/DM; (3) la búsqueda de los artículos se realizó en bases de datos como EBSCO, Science Direct, ProQuest y PsyArticles. Por último, las investigaciones experimentales revisadas debían haberse llevado a cabo en población adulta joven (20-40 años).

\section{La toma de decisiones desde un punto de vista neurofisiológico}

Se entiende por toma de decisiones (TD) el procesamiento de carácter cognitivo que realiza una persona cuando se encuentra en una situación en la que debe evaluar una o más características para establecer cuál de las alternativas cumple con sus expectativas, metas o intereses, de las cuales se debe derivar un proceso reflexivo o una conducta que se va a seguir (Wang, 2008).

La TD es un proceso complejo en el cual intervienen diferentes tipos de conexiones neuronales que actúan de manera interactiva, por lo que no se puede hablar de un proceso lineal del procesamiento de la información, sino de diferentes tipos de decisiones que conforman, neuronalmente, vías de respuesta que no se han determinado de modo pleno (Pearson \& Platt, 2012).

En modelos animales, la TD se ha relacionado con la actividad de la corteza prefrontal (CPF) y la porción posterior del lóbulo parietal. Consistentemente, en estudios con humanos se han relacionado las mismas áreas cerebrales con la TD (Gold \& Shalden, 2007; Wang, 2008, 2012).

Al evaluar la TD por medio de neuroimágenes a partir de diferentes actividades como valued-based decision y perception task en humanos y en primates por medio de estudios electrofisiológicos durante el desarrollo de actividades de memory-guided saccade task, se encontró que la corteza orbitofrontal, así como los ganglios basales, está asociada con el comportamiento de búsqueda de recompensa (Gold \& Shalden, 2007; Kawagoe, Ta- kikawa \& Hikosaka, 2004; Watanabe, Lauwereyns \& Hikosaka, 2003).

Otras investigaciones han concluido que algunas neuronas de la corteza orbitofrontal y de la porción ventromedial del CPF son responsables de atribuir un valor independiente a cada alternativa y participan en la decisión y acción correspondientes a la TD (Hunt, Kolling, Soltaní, Woolrich, Rushworth \& Behrens, 2012; Padoa-Schioppa \& Assad, 2006). Adicionalmente, la porción cingular anterior del CPF se ha relacionado con la evaluación negativa de una alternativa (Yeung \& Sanfey, 2004).

Hunt et al. (2012) han establecido, por medio de una combinación entre imágenes obtenidas por magnetoencefalograma (MEG), que la ruta de la TD en tareas simples inicia en la corteza visual, pasa por la corteza frontotemporal y por la ventromedial. De allí, la información se transmite al área medial y lateral de la corteza parietal para, finalmente, concluir en la respuesta motora. La elección de una alternativa no solo se relaciona con la información de la situación, sino a su vez con la historia de selecciones pasadas y las consecuencias (Barraclough, Conroy \& Lee, 2004; Sugrue, Corrado \& Newsome, 2005).

Además, Philiastides, Auksztelewicz, Heekeren \& Blankenburg (2011) mostraron resultados en los que la integración de evidencia de los estímulos es relevante en el momento de tomar decisiones de tipo perceptual, pues a partir de neuroimágenes se determina la participación del CPF dorsolateral en este proceso.

El estudio de la TD también se ha relacionado con diferentes variables como la personalidad y las decisiones grupales (Ahmed, Hasnain \& Venkatesan, 2012; Muehfeld, Doorn \& Witteloostuijin, 2011), manejo de la TD en grupos con dolor crónico (Fenwick, Chaboyer \& St. John, 2012) o mujeres diagnosticadas con cáncer de seno (Swainston, Campbell, Wersch \& Durning, 2012).

En el plano individual, diversos estudios han mostrado la forma en que las personas toman 
decisiones simples, por ejemplo, reproducir o no una canción (Major, 2013), la elección de productos teniendo las mismas cualidades físicas (Lysonski \& Durvasula, 2013; Zolfani, Rezaeiniya, Pourhossein \& Zavadskas, 2012), la preferencia de hombres para elegir entre las mujeres o el dinero (Jianmin, Yujiao \& Qinglin, 2012), la relación entre el envejecimiento y la TD (Brand \& Markowitsch, 2010) y la relación entre estrés y TD (Thompson, 2010).

\section{El estrés desde el punto de vista neurofisiológico}

Desde el punto de vista psicológico, el estrés es una respuesta desencadenada ante una demanda o situación potencialmente peligrosa que excede la capacidad del organismo para responder adecuadamente, lo cual provoca una respuesta fisiológica para compensar o responder a tales demandas del ambiente (Koolhaas et al., 2011).

La percepción del estresor desencadena repuestas fisiológicas que buscan preparar al organismo para enfrentar las demandas ambientales. Tales respuestas están reguladas por el sistema eje hipotalámico-hipofisario-adrenal (HHA), el cual es una red compleja a la cual se le atribuye el control de las reacciones al estrés por medio de la liberación de neurotransmisores, esteroides y péptidos que cumplen funciones de acuerdo con el tipo de estrés y con la prolongación de la situación estresante (Joëls \& Baraman, 2009).

La estructura activadora del HHA es el hipocampo, y como resultado de esta activación se liberan diferentes hormonas; así mismo, el hipocampo regula la liberación de hormonas de la hipófisis, las cuales se han relacionado con la respuesta al estrés como el cortisol y, en general, los glucocorticoides. Resultado de la liberación hormonal producida por el HHA, el hipocampo es inhibido (Cook \& Wellman, 2004) y así limita la capacidad de dicha estructura para detener la respuesta hormonal del HHA y como consecuencia las hormonas actúan de manera prolongada en el sistema nervioso. Este fenómeno se conoce como la hipótesis de la cascada de glucocorticoides (Bruce \& McEwen, 2007).

Las estructuras cerebrales que se afectan negativamente por la continua exposición al estrés son el hipocampo, la amígdala y el CPF, en las cuales se produce una pérdida de las conexiones dendríticas entre estas áreas (Fuchs, Flugge \& Czeh, 2006; Cook \& Wellman, 2004).

Por esta causa, el estrés agudo puede llegar a generar cambios negativos en las redes neuronales, de modo que una exposición prolongada a una situación estresante en el hipocampo es capaz de producir cambios en las dendritas del CA 3 de las neuronas piramidales y en la reducción del número de sinapsis (Kole, Czeh \& Fuchs, 2004). También se ha documentado que la exposición a estrés crónico en el CPF retrae las dendritas y hace que se pierdan espinas de las neuronas piramidales (Fuchs et al., 2006; Radley et al., 2004).

Igualmente, Pruessner et al. (2008) llevaron a cabo una investigación en la cual, por medio de neuroimágenes obtenidas de 40 participantes, llegaron a la conclusión de que la exposición a tareas estresantes por periodos relativamente largos (estrés agudo) ocasiona cambios metabólicos en las áreas prefrontal y límbica, en los ganglios basales, entre otros.

El estrés agudo, como lo menciona Arsten (2009), puede llegar a causar una rápida y drástica pérdida de las habilidades cognitivas directamente relacionadas con el CPF, donde la exposición prolongada a uno o a varios elementos estresantes puede tener consecuencias estructurales y, por ende, funcionales; así, las decisiones tomadas bajo condiciones estresantes se ven moduladas por el efecto del estrés en el área dorsolateral del CPF.

Las investigaciones demuestran que las regiones que están asociadas con la TD son sensibles al efecto que produce el estrés, por lo que se apoya la hipótesis de que el estrés impacta negativamente en la TD y también altera negativamente la capacidad de procesar información recibida del medio ambiente (Starcke \& Brand, 2012). 


\section{Estrés como factor limitante en la TD}

Además de centrarse en la relación de carácter fisiológico entre el estrés y la TD, las investigaciones han evidenciado el detrimento de la capacidad para elegir de manera exitosa cuando las personas se encuentran en un entorno percibido como estresante o que excede sus capacidades.

Kassam, Koslov y Méndez (2009) realizaron un experimento con 103 participantes (32 hombres y 71 mujeres) con una media de 22.4 años, quienes estuvieron expuestos al trier social stress, que consiste en una corta exposición de su perfil para ocupar un cargo (retroalimentada positiva o negativamente), seguida de diversas preguntas. Así, mientras los participantes se encontraban realizando esta prueba, se medía su presión arterial, frecuencia cardiaca y concentraciones de cortisol. Los resultados indicaron que el estrés producto de la exposición a un público evaluador y a la retroalimentación negativa evita que las personas respondan adecuadamente a la situación, por lo que el desempeño en las tareas se ve afectado de modo negativo.

Así mismo, Porcelli \& Delgado (2009), en su investigación con 27 personas (13 mujeres y 14 hombres), buscaban encontrar la relación existente entre un evento estresor extrínseco y la TD financieras por medio de juegos en los que debían elegir entre dos opciones, las cuales los llevaban a ganar o perder dinero. Simultáneamente, una de las manos era introducida en agua fría $\left(4^{\circ} \mathrm{C}\right)$ y ello funcionaba como estresor. Los resultados indicaron que estresores externos pueden alterar de manera significativa la TD y hacer que estas últimas se den de manera más conservadora, es decir, los participantes que tenían las manos en agua fría durante el juego no tomaron decisiones riesgosas y dejaron entonces de evaluar las opciones adecuadamente; así mismo, se observa que respondían de manera automática.

Por otro lado, Putman, Antypa, Crysovergi y van der Does (2010), con 29 estudiantes universi- tarios, intentaron establecer si tomaban o no más decisiones arriesgadas en presencia de factores estresantes. Los resultados en esta investigación indicaron que el estrés hace que las personas tomen decisiones más arriesgadas si la recompensa es alta, lo cual contradice de cierta manera la investigación de Porcelli \& Delgado (2009).

Teniendo en cuenta lo presentado, la literatura ha mostrado que el estrés afecta la capacidad que poseemos para responder adecuadamente a actividades que involucran la TD. Desde los aspectos neurofisiológicos, por el efecto que tiene en nuestro sistema nervioso una exposición prolongada a los glucocorticoides, que puede ser diferencial según la estructura cerebral que afecte; en el caso del CPF, área relacionada con la TD, se observa un impacto negativo en su funcionamiento, más específicamente en la búsqueda de recompensa, valoración negativa y valoración independiente de un objeto o situación dada. Y teniendo en cuenta aspectos comportamentales, las respuestas se toman basadas en el aprendizaje obtenido a partir del desempeño en situaciones previas, donde se experimentó estrés agudo.

\section{Diferencias de género en relación con el impacto del estrés en la toma de decisiones}

Existen diferencias significativas entre hombres y mujeres al responder a eventos estresantes. Golberg (2009) expone cómo la oxitocina, hormona que ante el estrés se ha relacionado con respuestas de calma y respuestas sociales positivas, es recaptada de manera diferente dependiendo del género. En situaciones de estrés, en las mujeres se han encontrado mayores concentraciones de oxitocina en comparación con los hombres. Desde el punto de vista conductual, esto implica que las mujeres ante eventos muy estresantes pueden responder de manera "pasiva" y prefieren encontrarse en un entorno donde puedan socializar lo ocurrido; mientras que los hombres pueden responder de manera 
más agresiva y tienden a aislarse para canalizar esas emociones.

Preston, Stansfield, Buchanan y Bechara (2007) encontraron que las mujeres responden de modo más adecuado ante una situación que genere estrés anticipatorio (discurso) que los hombres; sin embargo, durante la realización de las actividades estresantes los hombres tuvieron una mejor respuesta que las mujeres. Entonces, las mujeres tienen la capacidad de regular mejor sus respuestas fisiológicas y conductuales antes de que se presente la situación estresante y los hombres demostraron tener una mejor respuesta conductual y fisiológica ante la situación estresante. Por otro lado, se ha podido observar cómo los hombres que se encuentran bajo grados de estrés pueden llegar a aumentar la probabilidad de tomar decisiones riesgosas; mientras que las mujeres toman decisiones menos riesgosas (Van den Bos, Harteveld \& Stoop, 2009).

En la investigación de Lighthall et al. (2012) se encontró que las respuestas neuronales y comportamentales entre hombres y mujeres es opuesta: en los hombres hay una mayor elevación de las cantidades de cortisol que en las mujeres, y esto se debe a que, bajo condiciones estresantes, en los hombres hay una mayor activación del cuerpo estriado; mientras que en las mujeres decrece su activación. Esto lleva a que la velocidad de la respuesta sea más rápida $\mathrm{y}$, por ende, se da bajo un procesamiento más automático de la información en los hombres. A diferencia de la investigación realizada por Van den Bos et al. (2009), en esa investigación no se hallaron diferencias en la respuesta de riesgo entre género.

Así mismo, Schoofs, Pabst, Brand y Wolf (2013) encontraron que los hombres responden más rápidamente cuando se encuentran bajo estrés que sin estrés, contrario a las mujeres en el Trier Social Stress Test.

Wang et al. (2007) indican, además, que las diferencias halladas en las respuestas ante eventos estresores, tal como tener que realizar operaciones matemáticas ante un público y siendo presionado para que responda más rápido, se puede deber a la forma como se responde cerebralmente; de modo que mientras en las mujeres el flujo sanguíneo se incrementa en el sistema límbico y en el hemisferio izquierdo, en los hombres el flujo sanguíneo aumenta en el hemisferio derecho ante eventos altamente estresores. Estos hallazgos concuerdan con lo documentado por Golberg (2009).

Pabst, Brand y Wolf (2013) encontraron en una muestra de 40 hombres que el estrés afecta las estrategias utilizadas en el momento de evaluar una situación; así mismo, el proceso de ajuste de la persona cambia a una respuesta de manera automática, de modo que se ve inhibido el procesamiento de información. Por último, se observa en esta investigación que el análisis de la información retroalimentada por el ambiente no se tiene en cuenta cuando la persona está bajo niveles de estrés agudo, de tal manera que la persona continúa con un mismo patrón de respuesta. Pabst et al. (2013) encontraron que las personas, por falta de control de la situación y por el malestar que causa el encontrarse bajo estrés, buscan recompensas inmediatas. Esto tiene correlación con el mal funcionamiento del CPF que, de cierta manera, inhibe la gratificación inmediata y la TD riesgosas.

Finalmente, Van den Bos, Homberg y De Visser (2013), en una revisión teórica, proponen que las diferencias entre hombres y mujeres en la TD radica en la asimetría cerebral que hay entre los dos géneros; así que en las mujeres la irrigación sanguínea ante la TD se concentra principalmente en el hemisferio izquierdo, mientras que en los hombres se ha mostrado una mayor irrigación sanguínea en el hemisferio derecho. Esto, a su vez, puede relacionarse con el hecho de que los hombres se enfocan y deciden bajo información global que puedan tener de la situación y las mujeres se enfocan y deciden bajo información detallada de la situación. 


\section{Discusión}

Las evidencias teóricas presentadas en el documento confirman que existe una relación directa entre los niveles de estrés y la TD. A mayor estrés, menor es la capacidad de responder de manera efectiva a una demanda que requiera un procesamiento de información en el que se deban evaluar varias opciones para tomar una decisión confiable.

El estrés afecta neurofisiológicamente la capacidad para tomar decisiones de manera efectiva, en la medida en que los mecanismos o vías hormonales del estrés afectan las áreas relacionadas con la TD, como lo es la CPF. Según Wellman (2010), las dendritas de diferentes áreas cerebrales se ven afectadas físicamente por la exposición continua o prolongada al estrés, de modo que es posible que se pierdan o dañen determinadas conexiones sinápticas.

Ello lleva entonces a concluir que existe una relación negativa entre el estrés y la TD; que esta relación no solo provoca cambios comportamentales (irritabilidad, aislamiento o depresión), cognitivos (bloqueo, frustración, percepción de amenaza, baja autoestima y autoevaluación) y alteración en de las funciones fisiológicas (sistema endocrino y cardiovascular), sino que, a su vez, impacta estructuralmente en el cerebro. De esta forma, el estrés impacta el proceso de ajuste de las personas hacia la situación en la que se encuentran e impide que se tome una decisión estratégica, debido a la interferencia con el procesamiento de la información (Singer \& Chung, 2012). Otra variable que modifica la TD sería el género, de forma que características fisiológicas y funcionales particulares para cada género modulan las respuestas dadas en este ámbito.

Es necesario investigar la TD desde los diferentes tipos de decisiones que las personas debemos tomar. La mayoría de estudios se centran en TD a corto plazo y "simples", en la medida en que las decisiones que los sujetos han de tomar se resumen a oprimir algún botón en relación con una cantidad de dinero - BART, Game of Dice Task (GDT), Risky Gains Task, Cambridge Gampling Task-, evitar accidentes de tránsito - Driving Simulator \& Chicken Game - o realizar una exposición a un público evaluador - Trier Social Stress Test-.

\section{Referencias}

Ahmed, A., Hasnain, N., \& Venkatesan, M. (2012). Decision making in relation to personality types and cognitive styles of business students. The IUP Journal of Management Research, 11(2), 20-29.

American Psychological Association. (2009). Stress in America 2009. Recuperado de http://www. apa.org/news/press/releases/stress-exec-summary.pdf

American Psychological Association. (2012). Stress in America TM: Our health at risk. Recuperado de http://www.apa.org/news/press/releases/stress /2011/final-2011.pdf

American Psychological Association. (2013). Stress: The different kinds of stress. Recuperado de http://www.apa.org/helpcenter/stress-kinds. aspx

Arnsten, A. (2009). Stress signalling pathways that impair prefrontal cortex structure and function. Nature Reviwes Neuroscience, 10, 410-422. doi: $10.1038 / \mathrm{nrn} 2648$

Barraclough, D., Conroy, M., \& Lee D. (2004). Prefrontal cortex and decision making in a mixedstrategy game. Nature Reviews of Neuroscience, 7, 404-410, doi: 10.1038/nn1209

Brand, M. \& Markowitsch, H. (2010). Aging and decision-making: A Neurocognitive perspective. Gerontology, 56, 319-324. doi: $10.1159 / 000248829$

Bruce, S. \& McEwen, B. (2007). Physiology and neurobiology of stress and adaptation: central role of the brain. Physiology Review, 87, 873904. doi: 10.1152/physrev.00041

Cook, S. \& Wellman, C. (2004). Chronic stress alters dendritic morphology in rat medial prefrontal 
cortex. Journal of Neurobiology, 60, 236-248. doi: 10.1002/neu.20025

Fenwick, C., Chaboyer, W., \& St John, W. (2012). Decision-making processes for the self-management of persistent pain: A grounded theory study. Contemporary Nurse: A Journal for the Australian Nursing Profession, 42(1), 53-66. doi: 10.5172/conu.2012.42.1.53

Fuchs, E., Flugge, G. \& Czeh, B. (2006). Remodelling of neuronal networks by stress. Frontiers in Bioscience, 11, 2746-2758.

Golberg, E. (2009). The new executive brain: Frontal lobes in a complex world. New York: Oxford University Press.

Gold, J. \& Shadlen, M. (2007). The neural basis of decision-making. Annual Review of Neuroscience, 30, 535-574. doi:10.1146/annurev. neuro.29.051605.113038

Hunt, L., Kolling, N., Soltani, A., Woolrich, M., Rushworth, M., \& Behrens, T. (2012). Mechanisms underlying cortical activity during value-guided choice. Nature Reviews of Neuroscience, 15(3), 470-476. doi:10.1038/nn.3017

Jianmin, Z., Yujiao, W., \& Qinglin, Z. (2012). An ERP Study on Decisions between Attractive Females and Money. Plos ONE, 7(10), 1-8. doi:10.1371/ journal.pone. 0045945

Joëls, M. \& Baramans, T. (2009). The neuro-symphony of stress. Nature Reviews of Neuroscience, 10, 459-466.

Kassam, K. S., Koslov, K., \& Mendes, W. (2009). Decisions under distress: stress profiles influence anchoring and adjustment. Psychological Science, 20, 1394-1399. doi: 10.1111/j.14679280.2009.02455.x

Kawagoe, R., Takikawa, Y., \& Hikosaka, O. (2004). Reward-predicting activity of dopamine and caudate neurons - a possible mechanism of motivational control of saccadic eye movement. Journal of Neurophysiology, 91, 1013-1024. doi: 10.1152/jn.00721

Kole, M., Czeh, B. \& Fuchs, E. (2004). Homeostatic maintenance in excitability of tree shrew hippo- campal CA3 pyramidal neurons after chronic stress. Hippocampus, 14, 742-751. doi: 10.1002/ hipo.10212.

Koolhaas, J., Bartolomucci, A., Buwalda, B., de Boer, F., Flugge, G., Korte, M., ...Fuchs, E. (2011). Stress revisited: critical evaluation of the stress concept. Neuroscience \& Biobehavioral Reviews, 35, 1291-1130. doi: 10.1016/j.neubiorev

Leka, S., Griffiths, A., \& Cox, T. (2004). Work organization and stress: Systematic problem approaches for employers, managers and trade union representatives. Geneva: WHO.

Lighthall, N., Sakaki, M., Vasunilashorn, S., Nga, L., Somayajula, S., Chen, E., Samii, N., \& Mather, M. (2012). Gender differences in reward-related decision processing under stress. Social Cognitive and Affective Neuroscience, 7(4), 476-484. doi: $10.1093 / \mathrm{scan} / \mathrm{nsr} 026$

Lysonski, S. \& Durvasula, S. (2013). Consumer decision making styles in retailing: evolution of mindsets and psychological impacts. Journal of Consumer Marketing, 3(1), 75-87. doi: $10.1108 / 07363761311290858$

Major, M. (2013). How they decide: A case study examining the decision-making process for keeping or cutting music in a $\mathrm{K}-12$ public school district. Journal of Research in Music Education, 61(1), 5-25. doi: 10.1177/0022429412474313

Muehlfeld, K., Doorn, J., \& Witteloostuijn, A. (2011). The effects of personality composition and decision-making processes on change preferences of self-managing teams. Managerial \& Decision Economics, 32(5), 333-353. doi: 10.1002/ mde. 1539

Mujtaba, B., Lara, A., King, C., Johnson, V. \& Mahanna, T. (2010). Stress at work in a slowing economy. The Journal of Applied Management and Entrepreneurship, 15(2), 26-42.

Pabst, S., Brand, M. \& Wolf, O. (2013). Stress and decision making: A few minutes make all the difference. Behavioral Science Research, 250, 39-45. doi: 10.1016/j.bbr.2013.04.046 
Padoa-Schioppa, C. \& Assad, J. (2006). Neurons in the orbitofrontal cortex encode economic value. Nature (441), 223-226. doi:10.1038/ nature 04676

Pan American Health Organization. (2013). Causas principales de mortalidad en las Américas. Recuperado de http://ais.paho.org/phip/viz/ mort caus asprincipales It oms.asp

Pearson, J. \& Platt, M. (2012). Dynamic decision making in the brain. Nature Reviews of Neuroscience, 15(3), 341-343. doi:10.1038/nn.3049

Philiastides, M., Auksztelewicz, R., Heekeren, H., $\&$ Blankenburg, F. (2011). Casual role of dorsolateral cortex in human perceptual decision-making. Current Biology, 21, 980-983. doi: 10.1016/j.cub.2011.04.034

Porcelli, A. \& Delgado, M. (2009). Acute stress modulates risk taking in financial decision making. Psychological Science, 20, 278-283. doi: 10.1111/j.1467-9280.2009.02288.x

Preston, S., Stansfield, R., Buchanan, T., \& Bechara, A. (2007). Effects of anticipatory stress on decision making in a gambling task. Behavioral Neuroscience, 2, 257-263. doi: 10.1037/07357044.121.2.257

Pruessner, J., Dedovic, K., Khalili-Mahani, N., Engert, V., Pruessner, M., Buss, C., Renwick, R., Dagher, A., Meaney, M., \& Lupien, S. (2008). Deactivation of the limbic system during acute psychosocial stress: Evidence from positron emission tomography and functional magnetic resonance imaging studies. Biology Psychiatry, 63(2), 234-240.

Putman, P., Antypa, N., Crysovergi, P. \& van der Does, W. (2010). Exogenous Cortisol acutely influences motivated decision making in healthy young men. Psychopharmacology, 208, 257263. doi: 10.1007/s00213-009-1725-y

Radley, J., Sisti, H., Hao, J., Rocher, A., McCall, T., Hof, P., McEwen, B., \& Morrison, J. (2004). Chronic behavioral stress induces apical dendritic reorganization in pyramidal neurons of the medial prefrontal cortex. Neuroscience 125 , 1-6. doi: 10.1016/j.neuroscience.2004.01.006 Schoofs, D., Pabst, S., Brand, M., \& Wolf, O. (2013). Working memory is differentially affected by stress in men and women. Behavioural Brain Research, 241, 144-153. doi: 10.1016/j. bbr.2012.12.004

Singer, T. \& Chung, J. (2012). The perfect amount. Psychology Today, 79-85.

Starcke, K. \& Brand, M. (2012). Decision making under stress: A selective review. Neuroscience and Biobehavioral Reviews, 36, 1228-1248. doi:10.1016/j.neubiorev.2012.02. 003

Sugrue, L., Corrado, S., \& Newsome, W. (2005). Choosing the greater of two goods: neural currencies for valuation and decision-making. Nature Reviews of Neuroscience, 6, 363-375. doi:10.1038/nrn1666

Swainston, K., Campbell, C., van Wersch, A., \& Durning, P. (2012). Treatment decision making in breast cancer: A longitudinal exploration of women's experiences. British Journal of Health Psychology, 17(1), 155-170. doi:10.1111/ j.2044-8287.2011.02028.x

Thompson, H. (2010). The stress effect: Why smart leaders make dump decisions - and what do about it. San Francisco: Jossey-Bass A Wiley Imprint.

Van den Bos, R., Harteveld, M., \& Stoop, H. (2009). Stress and decision-making in humans: performance is related to cortisol reactivity, albeit differently in men and women. Psychoneuroendocrinology, 34, 1449-1458. doi: 10.1016/j. psyneuen.2009.04.016

Van der Bos, R., Homberg, J. \& De Visser, L. (2013). A critical review of sex differences in decision-making tasks: Focus on the Iowa Gambling Task. Behavioural Brain Research, 238, 95-108. doi: 10.1016/j.bbr.2012.10.002

Wang, J., Korczykowski, M., Rao, H., Fan, Y., Pluta, J., Gur, R., McEwen, B. \& Detre, J. (2007). Gender difference in neural response to psychological stress. Social Cognitive and Affective 
Neuroscience, 2(3), 227-239. doi: 10.1093/ scan/nsm018

Wang, X. (2008). Decision making in recurrent neuronal circuits. Neuron, 60, 215-234. doi: 10.1016/j.neuron.2008.09.034

Wang, X. (2012). Neural dynamics and circuit mechanisms of decision-making. Current Opinion in Neurobiology, 22, 1-8. doi: 10.1016/j. conb.2012.08.006

Watanabe, K., Lauwereyns, J., \& Hikosaka, O. (2003). Neural correlates of rewarded and unrewarded eye movements in the primate caudate nucleus. Journal of Neuroscience, 23, 10052-10057.

Wellman, C. (2010). Dendritic reorganization in piramidal neurons in medial prefrontal cortex after chronic corticosterone administration. Journal of Neurobiology, 49, 245-253. doi: 10.1002/ neu. 1079
Wiener, P. (2011). Neurotherapy in adult management of stress: Developmental aspects of the adults immune system. Journal of Adult Development, 18, 66-69.

Yeung, N. \& Sanfey, A. (2004). Independent coding of reward magnitude and valence in the human brain. Journal of Neuroscience, 24, 625-664. doi: 10.1523/jneurosci.4537-03.2004

Zolfani, S., Rezaeiniya, N., Pourhossein, M., \& Zavadskas, E. (2012). Decision making on advertisement strategy selection based on life cycle of products by applying FAHP and TOPSIS GREY: Growth stage perspective; a case about food industry in Iran. Engineering Economics, 23(5), 471-484. doi: http://dx.doi.org/10.5755/ j01.ee.23.5.3134
Fecha de recepción: 10 de julio de 2014 fecha de aceptación: 11 de mayo de 2015 